\title{
19p13.3 microduplication syndrome
}

INSERM

\section{Source}

INSERM. (1999). Orphanet: an online rare disease and orphan drug data base. 19p13.3 microduplication syndrome. ORPHA:447980

19p13.3 microduplication syndrome is a rare, genetic, syndromic intellectual disability characterized by intrauterine growth retardation, microcephaly, hypotonia, motor and neurodevelopmental delay, speech delay, intellectual disability, and mild dysmorphic features. 